Relations industrielles

Industrial Relations

\title{
Grievance Arbitration: a Review of Current Problems, ed. M.A. Hiclking, Vancouver, Institute of Industrial Relations, University of British Columbia, 1977, 190 pp.
}

\section{Rodrigue Blouin}

Volume 33, numéro 1, 1978

URI : https://id.erudit.org/iderudit/028855ar

DOI : https://doi.org/10.7202/028855ar

Aller au sommaire du numéro

Éditeur(s)

Département des relations industrielles de l'Université Laval

ISSN

0034-379X (imprimé)

1703-8138 (numérique)

Découvrir la revue

Citer ce compte rendu

Blouin, R. (1978). Compte rendu de [Grievance Arbitration: a Review of Current Problems, ed. M.A. Hiclking, Vancouver, Institute of Industrial Relations,

University of British Columbia, 1977, 190 pp.] Relations industrielles / Industrial Relations, 33(1), 156-159. https://doi.org/10.7202/028855ar

Tous droits réservés @ Département des relations industrielles de l'Université Laval, 1978
Ce document est protégé par la loi sur le droit d'auteur. L’utilisation des services d'Érudit (y compris la reproduction) est assujettie à sa politique d'utilisation que vous pouvez consulter en ligne.

https://apropos.erudit.org/fr/usagers/politique-dutilisation/ 
pays comme dans l'autre, que les problèmes sont identiques et, enfin, que ceux qui font des interventions judiciaires dans le forum où la clause privative le défend sont aussi habiles, inventifs et efficaces dans un pays comme dans l'autre.

Chapitre 13. Ce chapitre traite des différends juridictionnels entre divers métiers et divers emplois et fonctions. Ici encore, c'est en lisant ce qui se passe aux ÉtatsUnis que le juriste canadien en apprend autant que le juriste américain qui lirait ce même chapitre 13 .

Chapitre 14. Finalement, pourtant le soussigné aurait dû savoir que l'on en arriverait là, le dernier chapitre traite des affaires intrasyndicales, c'est-à-dire, les conflits entre le syndicat comme entité en soi et le membre ou groupe de membres en conflit avec leur syndicat.

Il n'y a pas l'ombre d'un doute que le membre a plus de protection légale vis-àvis son syndicat aux États-Unis qu'au Canada. Cependant, il est trop facile de s'arrêter après avoir étudié l'existence d'un droit dans un pays comparé avec un autre sans se référer à l'Histoire. Au Canada, si le membre a moins de protection légale, son syndicat a moins de protection légale, son syndicat a moins de pouvoir pour exercer une discipline et des pénalités affectant ses membres.

Le soussigné se doit d'attirer l'attention du lecteur à une sorte d'appendice intitulée «ENGLISH-FRENCH LABOR RELATIONS GLOSSARY». C'est là que l'on donne les traductions: "Agreement" (Convention collective); "Application" (Demande d'emploi.); «Bump» (Supplanter ou déplacer.); «Call-Back Pay» (Indemnité de rappel.); et de bien d'autres expressions que l'on trouve dans les relations ouvrières.

Il est peut-être malheureux que l'on n'ait pas référé nos confrères américains à l'ouvrage de l'auteur Gérard Dion, Dictionnaire canadien des relations du travail (Québec, Fondation Gérard DION et les Presses de l'université Laval, 1976.), qui est plus complet et plus recherché que ces quelques pages.
En tant qu'avocat consacré à la pratique, docteur en droit, juriste et auteur, le soussigné soumet humblement que ce livre mérite l'attention de tous ceux qui s'intéressent aux relations de travail au $\mathrm{Ca}$ nada.

Philip CUTLER, C.R.L.L.D.

Montréal

Grievance Arbitration: a Review of Current Problems, ed. M. A. Hiclking, Vancouver, Institute of Industrial Relations, University of British Columbia, 1977, 190 pp.

Selon les propos mêmes du professeur M. Thompson, "The papers in this collection represent analyses of the more difficult issues facing arbitrators and advocates in Western Canada». En fait, les textes sont avant tout axés sur le droit en vigueur en Colombie-Britannique. Ce recueil qui regroupe neuf articles, pour la plupart d'une qualité exceptionnelle, mérite d'être consulté par tous les intéressés à l'arbitrage des griefs et plus particulièrement par le lecteur québécois. Car si l'économie fondamentale de l'arbitrage des griefs reste à peu de chose près la même au Québec, en Colombie-Britannique et dans les autres provinces canadiennes, il n'en demeure par contre pas moins que les solutions apportées aux problèmes concrets présentent des variantes à l'occasion importantes.

Ainsi, dans un article intitulé "The Code, the Collective Agreement, and the Arbitration Process: as Seen from the Labour Board", monsieur P. C. Weiler, président de la Commission des relations du travail de la Colombie-Britannique, fait une excellente revue de synthèse de l'économie générale du système de l'arbitrage des griefs, système qu'il qualifie comme étant «a private system under the control of the parties themselves». Par ailleurs, monsieur Weiler mentionne que «the B.C. Labour Code takes a unique approach to the problem of administration of collective agreements, by contrast with other North American jurisdictions. It establishes a comprehensive statutory framework for 
this process. The ultime source of collective agreement law is now located in Part VI of the Code, and labours arbitrators are no longer bound by the common law of contract or of commercial arbitration». A ce sujet, qu'il nous soit permis de souligner que le caractère statutaire de l'arbitrage des griefs au Québec a été constaté à maintes reprises par la doctrine et la jurisprudence notamment par la Cour d'Appel. De même, les arbitres québécois ne sont détachés de l'emprise civiliste pour créer un système de règles à l'occasion empruntées, il est vrai, du droit civil ou du droit criminel mais adaptées et à l'occasion originales; des exemples ressortent en matière du fardeau et de la qualité de la preuve, des règles d'interprétation, etc. Par ailleurs, si l'on fait abstraction du fait qu'au Québec l'arbitrage des griefs est l'exclusif moyen retenu pour régler les problèmes d'administration de la convention collective alors qu'en Colombie-Britannique les parties peuvent prévoir une autre voie, les deux systèmes présentent de grandes analogies. On doit cependant noter un point crucial de différentiation: le rôle joué par la Commission des relations du travail. Ce rôle est double: intervention médiatrice pour aider les parties à trouver une solution à la mésentente (grief); une "supervisory jurisdiction». Concernant cette dernière fonction, monsieur Weiler s'attache à en délimiter les aires. Il s'agirait ni d'une véritable juridiction «en appel», ni d'une juridiction «en évocation», mais d'une juridiction permettant à la Commission «to ensure conformity by arbitrators to the principales of labour law». Les quelques pages consacrées à ce sujet méritent une attention particulière. La solution retenue par le législateur est originale et la façon dont la Commission a compris son rôle particulièrement appropriée.

On aura pu le constater, l'article de monsieur Weiler vaut une lecture attentive, tout comme d'ailleurs le texte qui le suit: «Work Now, Grieve Later» du professeur J. M. MacIntyre.

Les propos du professeur MacIntyre ont ceci de particulièrement intéressant qu'ils permettent de constater que la règle "Work Now, Grieve Later» reste un principe fondamental qui appelle cependant «some speculations and predictions about its application». Or, il importe de le signaler, les orientations suggérées par le professeur McIntyre ont déjà retenu l'attention des arbitres québécois. Dans la jurisprudence émanant de la Colombie-Britannique, à l'exemple des autres provinces, un salarié ne peut refuser d'obéir que si l'ordre donné est illégal ou comporte un danger sérieux pour sa santé ou sa sécurité. L'auteur procède à une synthèse du sens et de la portée de la règle ainsi qu'aux questions relatives à la preuve. Il peut être d'intérêt de signaler qu'une nouvelle exception, dite «Personal Appearance Exception», a à quelques occasions été signalée. Cette exception se fonde comme suit: "The basis of this rule, created by arbitrators, is that if the grievor follows the order and is later vindicated by arbitration, money will make him whole... (here) there is no way that one can assess the loss to that man or woman". En conclusion à son étude, le professeur McIntyre insiste sur le fait que les arbitres doivent prendre conscience «that the rule will never be absolute, but also to attempt to make grievance or arbitration more attractive than self-help and its eventual results». Concernant cette dernière remarque, on peut noter que l'approche suivie par les arbitres québécois peut être susceptible d'amener les salariés à croire davantage en l'efficacité de la convention collective et du système d'arbitrage de griefs. En effet, les arbitres du Québec ont donné une extension aux exceptions à la règle de "Work Now, Grieve Later» en reconnaissant qu'un salarié puisse être éventuellement justifié de refuser d'obéir à un ordre lorsque tel ordre est "prima facie" contraire aux prescriptions de la convention collective. En un tel cas, les arbitres étudient le grief en s'en rapportant aux prescriptions objectives de la convention collective plutôt que de se restreindre à se demander comme ce semble le cas dans les autres provinces, si l'ordre donné pouvait amener le salarié à commettre un geste illégal ou ayant des conséquences sur la santé ou la sécurité ou encore, si l'ordre donné était tel que "The adequacy of the Grievance Procedure» n'existe pas.

Suivent le texte du professeur McIntyre, des articles du professeur J. M. Weiler et de Me. R. B. Bird. Le sujet traité par le 
premier s'intitule «Non-culpable Cause for Discharge: A New Perspective». Il s'agit d'un texte bien construit où l'auteur constate que la relation de travail peut être rompue lorsqu'un salarié ne peut plus, en raison de maladie ou d'autres causes analogues, assumer ses obligations. Le professeur Weiller fait état des tendances jurisprudentielles, d'expériences de droit comparé, des fondements aux approches retenues. Au sujet de l'orientation jurisprudentielle de principe, l'auteur fait une observation valable pour le Québec: "But we must understand that with this latter approach comes the implication that parties are refusing to take any responsability for the person if he becomes unemployable and, in the absence of any agreement to the contrary, they are recognizing the responsability is solely of a public nature to be dealt with by the legislatures. The issue then becomes whether they are content with the legislative response or, to be more accurate in reflecting the situation in this country at least, the lack of any legislative response».

En ce qui concerne le texte de Me Bird, "Substitution of Penalties under the Labour Code of B.C.», il s'agit d'une synthèse des principales décisions en la matière, synthèse qui n'est pas limitée à la jurisprudence arbitrale de la ColombieBritannique. On notera que la législation récente semble avoir enlever aux parties la possibilité de prévoir des peines spécifiques à l'égard de certains actes fautifs: "Whether this means the parties cannot by agreement oust the jurisdiction to review and substitute penalties has not been determinated». Il est à espérer que le législateur québécois saura tenir compte de cette expérience dans ses prochaines modifications touchant l'arbitrage des griefs.

L'étude de Me Bird est suivie d'un article sur les "Probationary Employees" de Me D. L. Larson. L'auteur y étudie plus particulièrement la possibilité pour les employés en période de probation de faire vérifier le bien-fondé de la non-continuité du lieu d'emploi. En conclusion, à sa synthèse particulièrement intéressante, $\mathrm{Me}$ Larson avance «that the preoccupation of arbitrators in relation to probationary employees will be to develop the concept of "suitability" as it finds itself into more and more collective agreements. In that way, the term perhaps will become as familiar as that other arbitral mainstray", "proper cause». On notera ici un contexte qui a peu de similitude avec celui du Québec mais où il y aurait cependant avantage à puiser.

Deux textes, «Promotion and Seniority» de Me B. Williams et "Loss of Seniority and the Nature of Seniority Rights" du professeur L. C. Smith apparaissent à la suite de l'étude de Me Larson. Ces deux articles attestent de l'importance de la notion d'ancienneté dans notre système de relations du travail et des difficultés d'application qu'elle soulève, plus particulièrement à l'égard de la notion de compétence. À noter l'expression «arbuspruderce» utilisée par Me Williams. Par ailleurs, il convient d'attirer l'attention sur l'exposé du professeur Smith à propos des trois approches qui ont été développées en milieu américain relativement aux droits d'ancienneté : "Status Quo» or "Follow the White Man» approach; the «Rightful Place» approach; the "Freedom Now» theory. Ces deux articles intéresseront sans nul doute le lecteur québécois car il comble un vide dans notre littérature.

Enfin, un article intitulé "The Admission and Use of Extrinsic Evidence in Labour Arbitrations ", signé par le professeur P. A. Gall et monsieur D. Jordan, ainsi qu'un texte de monsieur J. Baigont, vice-président du L.R.B. of B. C. et portant sur "The Labour Relations Board and Arbitration Process Under the New Provisions of the Labour Code", complètent le recueil. Dans le premier article, les auteurs analysent l'admissibilité en preuve des documents autres que la convention collective, $\mathrm{y}$ incluant des pièces attestant l'histoire des négociations. Il semble que «all extrinsic evidence is admissible if introduced for the purposes of shedding light on the shedding light on the meaning to be quen to the contractual language». Au Québec, une telle preuve ne serait admissible qu'en présence d'une ambiguité manifeste de la convention collective.

En ce qui concerne le texte de monsieur Baigont, il s'inscrit dans le prolongement des propos faits par monsieur P. X. Weiler. Monsieur Baignont présente une synthèse bien articulée des rôles respectifs 
de l'arbitrage des griefs et de la Commission des relations du travail. $\AA$ noter ici aussi l'insistance de monsieur Baigont pour bien situer la «supervisory jurisdiction» de la Commission: «(...) when an arbitration award is arrived at in a manner inconsistent with the procedure and principles mandated by the code, then this Board will not hesitate to insinuate itself into the arbitration process. When that mandate has been carried out then the fact that this board does not agree with the arbitrator's interpretation of the collective agreement, is no grounds for a reversal under section 108 of the Code".

En définitive, "Grievance Arbitration: A Review of Current Problems", est un ouvrage qui ne peut être ignoré par aucun intéressé à l'arbitrage des griefs. Pour le lecteur québécois, il apparaîtra certes que les solutions qui y sont rapportées ne sont pas toutes applicables ici, ni même importantes en totalité. Néanmoins, la majorité de ces articles aidera notre doctrine et notre jurisprudence si ces textes sont utilisés avec circonspection. À ce sujet, mentionnons que l'utilisation irréfléchie de solutions adoptées dans un contexte socioéconomique bien particularié et en égard de conventions collectives qui traduisent l'état d'évolution de ce contexte socio-économique, ne pourrait que créer des difficultés de fonctionnement dans notre propre système de relations du travail.

Rodrigue BLOUIN

Université Laval

The Practice of Industrial Relations, by D. A. Peach and D. Kuechle, Toronto, Mc-Graw-Hill Ryerson Ltd., 1975, $358 \mathrm{pp}$.

Ce volume ne peut être considéré comme un exposé systématique du système des relations industrielles. L'ouvrage entend donner un enseignement de la pratique des relations du travail au Canada et aux États-Unis et se veut avant tout destiné au personnel de la gérance et aux étudiants en sciences administratives.

La méthode utilisée par les auteurs est descriptive et généralement complétée par des cas. Dix thèmes, axés exclusivement sur le processus de la négociation collective, ses éléments et ses résultats, sont présentés. Au tout début, on retrouve une introduction de circonstance (thème 1) et une traditionnelle histoire des relations du travail (thème 2). Une présentation de la législation constitue l'objet du troisième thème: cette présentation attache une énorme importance à l'influence américaine. Le quatrième thème, intitulé «Union Organisation", est en fait consacré aux principes de base gouvernant l'accréditation et la négociation entre les parties. Suivent des thèses relatant la théorie et la pratique de la négociation collective (thème 5) et de la médiation (thème 6). Le septième thème porte sur la convention collective, qualifiée de "contract administration", tandis que le huitième thème décrit l'arbitrage des griefs. Le syndicalisme dans la fonction publique fait l'objet du neuvième thème. Enfin, le dernier thème entend aider à la mise en place d'un système valable de relations du travail dans une entreprise.

De façon générale, cet ouvrage se lit bien. Par contre, celui qui a déjà une compréhension de base du système des relations industrielles tirera plus de profit de la lecture que le débutant. Deux observations d'ordre général nous apparaissent nécessaires à ce sujet. En premier lieu, nous estimons que le "système des relations industrielles » ne peut être réduit aux seules relations du travail. Les relations industrielles se préoccupent par exemple, selon notre entendement, des problèmes de la main-d'œuvre (marché du travail, emploi, etc.). De plus, les relations collectives ne forment pas la matière exclusive du champ des préoccupations. On ne peut ignorer par exemple l'apport des normes étatiques du travail. Or, dans le volume, ces diverses considérations sont presqu'ignorées. Ainsi, le débutant risque de percevoir les relations industrielles d'une façon limitative. Il aurait convenu, selon notre opinion, que l'on fasse mention des grandes théories des diverses approches et des auteurs éminents qui ont façonné les relations industrielles. Par ailleurs, il aurait été préférable que certaines considérations qui nous semblent étrangères au système des relations industrielles, soient ignorées. À titre d'exemple, 